\title{
Simultaneous and multisite measure of micromotion, subsidence and gap to evaluate femoral stem stability
}

\author{
Michael Gortchacow ${ }^{a}$, Michael Wettstein ${ }^{b}$, Dominique P. Pioletti ${ }^{a}$, Magdalena Müller-Gerbl ${ }^{c}$, \\ Alexandre Terrier ${ }^{\mathrm{a}, *}$ \\ a Laboratory of Biomechanical Orthopedics, Ecole Polytechnique Fédérale de Lausanne, Station 19, 1015 Lausanne, Switzerland \\ ${ }^{\mathrm{b}}$ Service of Orthopaedics and Traumatology, University Hospital Center and University of Lausanne, Rue du Bugnon 46, 1011 Lausanne, Switzerland \\ ${ }^{\mathrm{c}}$ Department of Biomedicine, Institute of Anatomy, University of Basel, Switzerland
}

\section{A R T I C L E I N F O}

Article history:

Accepted 29 January 2012

\section{Keywords:}

Micromotion

Subsidence

Gap

Arthroplasty

Hip

Micro CT

\begin{abstract}
A B S T R A C T
The initial stability of cementless femoral components is crucial for the long-term success of total hip arthroplasty. This has been reported in animal and clinical studies. Until now, the stability was evaluated by the measurement of relative micromotion on a few simultaneous locations around the stem in cadaveric experiments. This paper presents an extended experimental setup to measure simultaneously local micromotion, subsidence and gap on hundreds of points at the bone-stem interface. This technique we applied to anatomical and straight stems in three pairs of cadaveric femurs. Measurements were in agreement with typically reported values. Conversely to other methods, which measure micromotion between implant and bone anchoring points of the measuring device, our method provides local micromotion between stem surface and adjacent bone surface. The observed variation of micromotion at the peri-implant surface confirms the importance of this simultaneous measure on a lot of points around the implant.
\end{abstract}

(c) 2012 Elsevier Ltd. All rights reserved.

\section{Introduction}

In young patients, cementless total hip replacement is actually often preferred to cemented stems because the use of an additional dead foreign body is avoided (Hozack et al., 1993). Although the survival rate at 15 years of some cementless stems is lower than cemented ones, other cementless designs show equal results (Flecher et al., 2010; Mäkelä et al., 2010; Merle et al., 2010). Failure is mainly associated to aseptic loosening, and has been related, among other problems, to primary stability in animal and human studies (Pilliar et al., 1986; Engh et al., 1992; Bragdon et al., 1996; Jasty et al., 1997). A critical value of $150 \mu \mathrm{m}$ of micromotion has been reported as a maximal limit to avoid fibrous tissue formation instead of bone ingrowth (Jasty et al., 1997). Besides, ample evidence indicates that mechanical cues affect the apposition of bone by osteoblastic cells as well as the osteoblastic differentiation pathway (Riddle and Donahue, 2009). Therefore, the primary stability is the most accepted mechanical quantity associated to the long-term success of cementless stems (Callaghan et al., 1992).

\footnotetext{
* Corresponding author. Tel.: +4121693 9498; fax: +41216938660.

E-mail address: alexandre.terrier@epfl.ch (A. Terrier).
}

Different techniques have already been proposed to measure bone-implant micromotion after cementless total hip replacement. This measure is usually divided into subsidence (Steimer et al., 2006) and micromotion (Baleani et al., 2000). Recent studies are mainly using linear variable differential transducers (LVDT's) (Walker et al., 1987; Gilbert et al., 1992; Buhler et al., 1997; Britton et al., 2004; Cristofolini et al., 2007; Gheduzzi and Miles, 2007. LVDT however do not really measure the local relative micromotion between the stem and the bone, but also include bone deformation between the device fixation and the measurement site. Besides, the number of simultaneous measurement points is limited to 1 or 2 , with a reported maximum of 4 (Cristofolini et al., 2003). However, one or two values of micromotion cannot characterize the complex mechanical environment of the peri-implant tissue. Recently, a new technique was proposed to measure micromotion, locally and at multiple sites simultaneously (Gortchacow et al., 2011). This method was based on micro-CT imaging of loaded cadaveric femurs, with a metallic stem and radio-opaque beads. This short communication reported only 8 simultaneous measurement sites on a single femur, and did not estimate the bone-implant gap.

Therefore, the goal of this study is twofold: first, we wanted to extend a cadaveric setup for measuring not only micromotion and subsidence (Gortchacow et al., 2011) but also peri-implant gap, to estimate the interstitial strain. In a second step, we aimed to 
apply this new technique to compare the primary stability of an anatomical versus a straight stem design, which are based on a different stability concept (Callaghan et al., 1992).

\section{Materials and methods}

\subsection{Prostheses}

We used two cementless femoral stems designs: anatomical and straight. The anatomical design is adapted to the anatomical shape of the femur, while the straight design has a rectangular cross-section shape. Three anatomical (SPS) and straight (Harmony) stems were provided by the manufacturer (Symbios Orthopédie SA, Switzerland). Both are made of titanium alloy (Ti6Al4V). The SPS is bicoated with porous titanium and hydroxyapatite and the Harmony is coated with hydroxyapatite. Both were fully coated.

\subsection{Cadaveric femurs}

Six human femora from 3 cadavers were used. The donors were 92, 88 and 87 year old females. The femurs were fixed in a $4 \%$ formalin solution. A classical 2D pre-operative planning was performed by a senior orthopedic surgeon with X-rays images of all the femurs for both prosthetic designs. No sign of pathology was observed on the X-rays contraindicating the implantation of a stem. An anatomical and straight stem was implanted for every pair of femurs. The selection between right and left for a given stem design was random.

\subsection{Experimental setup}

A senior surgeon achieved the pre-operation planning for each stem according to manufacturer recommendations. Before implantation of the stems, 12 Tantalum markers of $800 \mu \mathrm{m}$ in diameter were placed on the stem surface and 500 stainless steel markers of $600 \mu \mathrm{m}$ in diameter were placed on the reamed medullar cavity. The tantalum markers (stem markers) were dilled into the stem surface. The steel markers (bone markers) were distributed as uniformly as possible and press fit to bone with a spatula. Each stem with its markers was scanned by a $\mu$ CT (1076 Skyscan, Belgium) at a resolution (voxel size) of $36 \mu \mathrm{m}$, to get a reconstruction of its surface and location of its markers. This geometric reconstruction was done with the imaging software Amira (www.amira.com). Then, the surgeon completed the final implantation of the stem. After implantation, the femoral condyles were resected at a distance of approximately $220 \mathrm{~mm}$ from the greater trochanter. The distal end of the femur was cemented (Neukadur Multicast 20, Altropol Gmbh, Germany) into a cylindrical cup (Fig. 1A). We used the cylindrical cup as a guide to guarantee the same alignment for each stem. The stem neck was cut with a
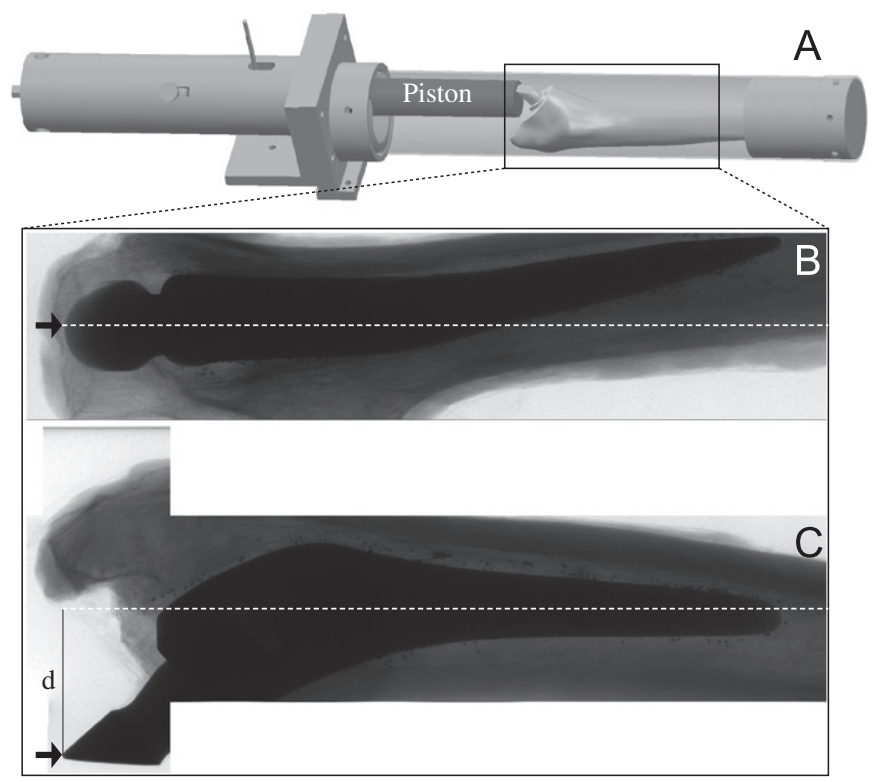

Fig. 1. Experimental setup with the loading device that is designed to be inserted into the $\mu \mathrm{CT}(\mathrm{A})$. Anteroposterior (B) and mediolateral (C) scout view from the $\mu \mathrm{CT}$ of a femur and stem mounted on the loading. The vertical dotted line represents the center axis of the loading device and the arrow indicates loading point of the piston. diamond saw so as to set the loading point at the center of the femoral head (Fig. 1B, C). A load was applied at the femoral head center with a piston. This loading device was designed to operate within the $\mu \mathrm{CT}$. The center of mass of all markers was localized using a home-made algorithm (Gortchacow et al., 2011). Four successive $\mu \mathrm{CT}$ scans were performed for all stems: a 1st scan without load, a 2nd scan with a load of $2000 \mathrm{~N}$, a 3rd scan without load and a 4th scan without load.

\subsection{Peri-implant micromotion, subsidence and gap}

Stem markers were used to determine the global position of the stem (Fig. 2A), while bone markers were used to determine the local displacement of bone (Fig. 2B). Stem markers defined a frame of reference fixed to the stem. This reference frame was defined at the 3rd unloaded case. It was obtained by a rigid body transformation of the stem markers (Gortchacow et al., 2011). Local micromotion was defined as the local displacement of bone markers in the loaded case (2nd scan) using the stem reference frame (3rd scan). Micromotion was divided into a tangential and perpendicular component relative to the surface of the stem. Subsidence was obtained from the position of bone markers in the initial unloaded case (1st scan) using the stem reference frame (3rd scan). We thus get the local displacement of bone after loading and unloading of the stem. Periimplant gap was calculated as the closest distance between the (reconstructed) stem surface and bone marker in the reference frame.

\subsection{Measurement error}

The measurement error was evaluated with two subsequent unloaded scans (3rd and 4th scans). The loading device was randomly moved within the $\mu \mathrm{CT}$ to have a different position between these two unloaded scans. The error of micromotion (and subsidence) was obtained by measuring the displacement of the bone markers of the fourth unloaded scan in the stem reference frame (third scan). The distribution of this displacement in each spatial coordinate $(x, y$ and $z)$ followed a normal distribution with mean 0 and standard deviation $\sigma$. We defined the error as the $95 \%$ predictability band (prediction interval) of a single measurement along the axis with the greatest error, i.e. $1.96 \operatorname{Max}\left(\sigma_{x}, \sigma_{y}, \sigma_{z}\right)$, following the method of a previous technical paper (Gortchacow et al., 2011).

\subsection{Strain}

We assumed that octahedral shear strain $\gamma_{\text {oct }}$ is the mechanical variable that drives the cell differentiation, which produces fibrous tissue when $\gamma_{\text {oct }}$ is greater than a critical value 0.1125 . This value was estimated from another tissue differentiation model (Huiskes et al., 1997), assuming that there is no fluid flow. We also verified that this value of $\gamma_{\text {oct }}$ was within the range of reported values for fibrous tissue formation (Soballe et al., 1993). The octahedral stain was derived from its general definition

$\gamma_{\mathrm{oct}}=\frac{2}{3} \sqrt{\sum_{i \neq j}\left(E_{i}-E_{j}\right)^{2}}$

where $E_{i}$ are the eigenvalues of the finite stain tensor $E$. Assuming that bone and implant surfaces are parallel and rigid (compared to the soft tissue) in the vicinity of the bone marker, this strain measure of bone was approximated (only quadratic terms) with the following expression

$\gamma_{\text {oct }}\left(\delta_{t}, \delta_{n}\right)=\frac{1}{3} \sqrt{2} \sqrt{\delta_{n}^{2}\left(2+\delta_{n}\right)^{2}+\left(3+2 \delta_{n}\left(2+\delta_{n}\right)\right) \delta_{t}^{2}+\delta_{t}^{4}} \cong \frac{1}{3} \sqrt{2} \sqrt{4 \delta_{n}^{2}+3 \delta_{t}^{2}}$

where the dimensionless quantities $\delta_{n}=\delta h_{n} / h$ and $\delta_{t}=\delta h_{t} / h$ are defined from the perpendicular $\delta h_{n}$ and tangential $\delta h_{t}$ components of the micromotion at the stem surface, and $h$ is the gap between the bone marker and the stem surface (Fig. 2).

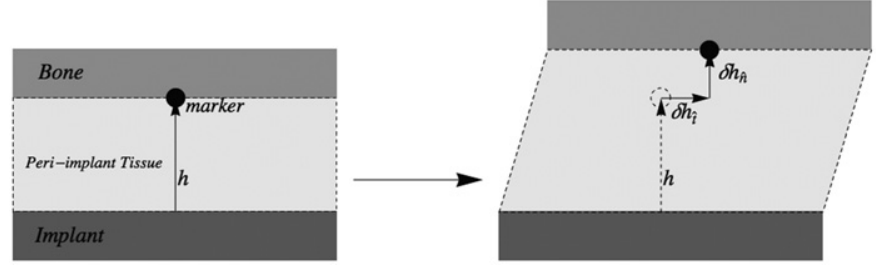

Fig. 2. In the vicinity of the marker, the bone and the implant surfaces are approximted as rigid, parallel and separated by a gap $h$ (left). When a load is applied, the peri-implant tissue strain can be caraterized by the gap $h$ and perpendicular $\delta h_{n}$ and tangential $\delta h_{t}$ micromotion (right). 


\subsection{Data analysis}

Micromotion, subsidence, gap and strain measurements were grouped in 4 usual anatomical sides: anterior $(\mathrm{A})$, posterior $(\mathrm{P})$, medial $(\mathrm{P})$ and lateral $(\mathrm{L})$. The medial quarter was aligned with the direction defined by the stem center and the application force point. The other sides were set according to the medial one. For each side, boxplots indicate median, 2 extremes, 2 nd quartile (25\% of data under the median), 3rd quartile (25\% of the data over the median) and number or markers.

\section{Results}

Local micromotion, subsidence and gap in the 3 directions were successfully obtained for the 6 tested stems. The amount of markers within each side varied between 7 and 80 .

\subsection{Micromotion and subsidence}

The micromotion error was $\pm 15 \mu \mathrm{m}$ and approximately equal in all directions. Overall, micromotion was below $150 \mu \mathrm{m}$, except for the SPS of pair \#1 and the SPS of pair \#3 (Fig. 3). The fraction of markers above the critical limit was $11 \%$ for the SPS of pair \#1 and $14 \%$ for the SPS of pair \#3. The average and spread of the micromotion was globally lower for the Harmony. In pair \#1, the
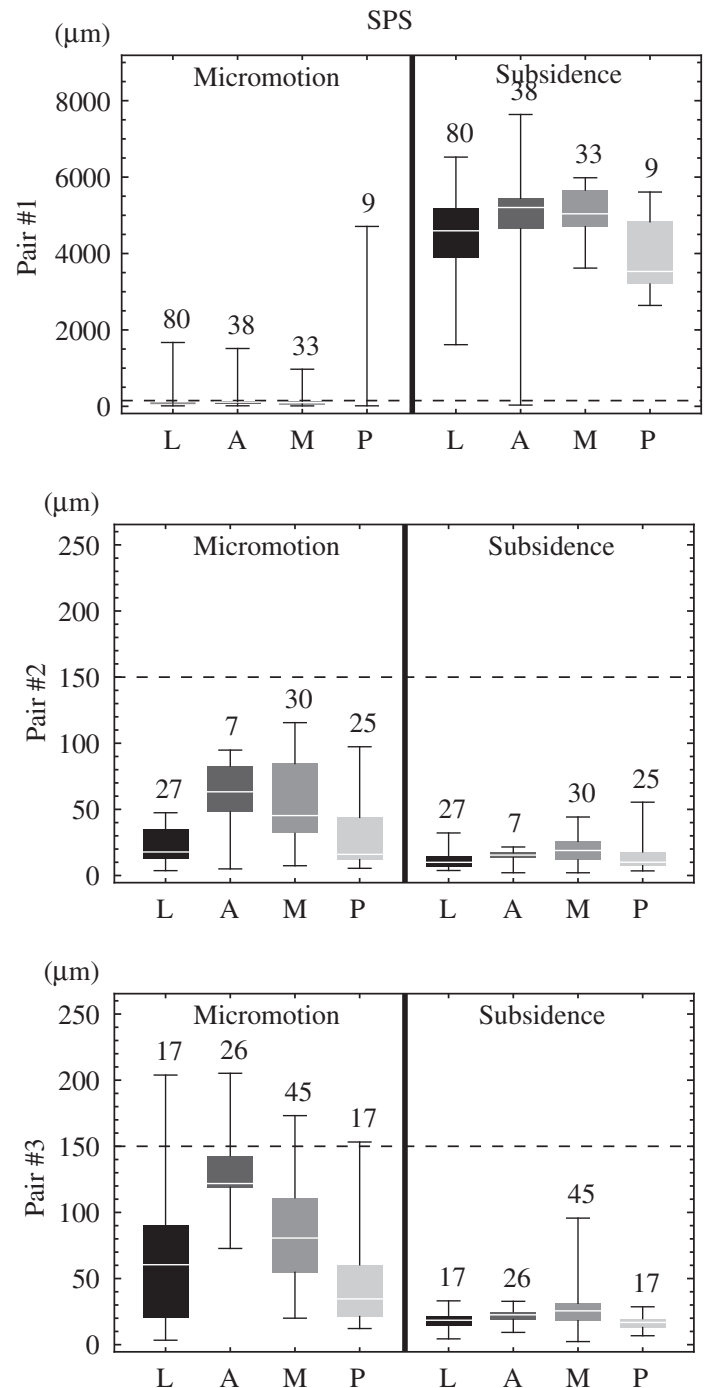

subsidence was nearly $5 \mathrm{~mm}$ in the SPS, while there was only a slight subsidence in the Harmony (Fig. 3). Actually, this case showed lowest subsidence. In pair \#2, both stems performed very similarly. The micromotion was below the critical limit and there was no difference in subsidence. Pair \#3 was rather similar than pair \#2, except in the anterior region, where micromotion was higher for the SPS. Overall, the SPS was less stable for this pair.

\subsection{Gap}

The bone-implant gap ranged between 0 and $7000 \mu \mathrm{m}$ (Fig. 4). For all femurs, the median was between 500 and $2000 \mu \mathrm{m}$. There was not apparent relationship between gap, micromotion, subsidence, stem types or measurements sides.

\subsection{Strain}

Strain was very different from the micromotion (Fig. 5). In pair \#1, fraction of markers above the strain limit was $28 \%$ for the SPS, while it remained below the critical value $\gamma c_{\text {oct }}$ for the Harmony. Conversely, in pair \#2, the strain fraction was $2 \%$ for the SPS and 7\% for the Harmony. In pair \#3, the strain was similar to pair \#2, with a fraction of $19 \%$ for the SPS and $1 \%$ for the Harmony. Overall,

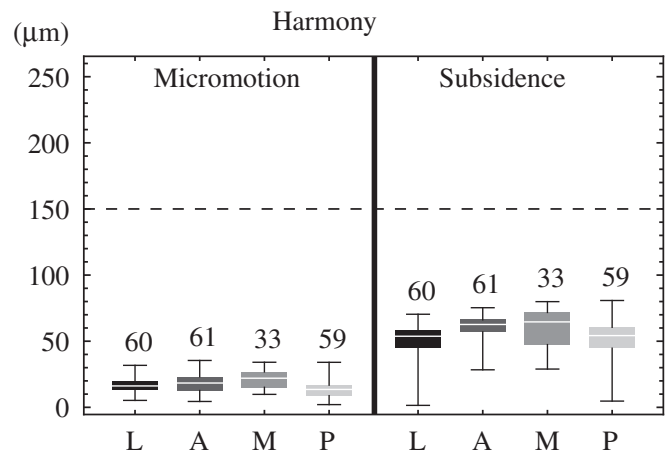

$(\mu \mathrm{m})$

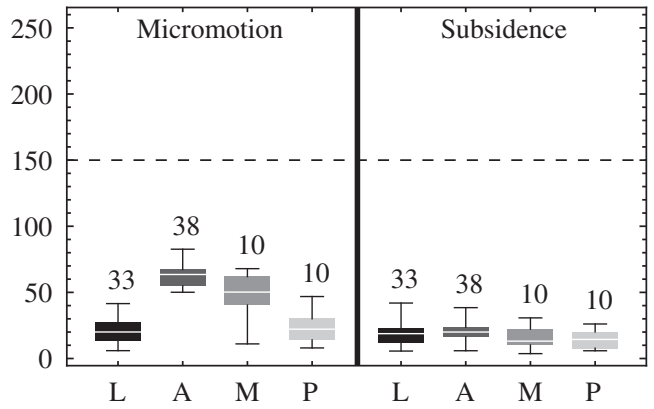

$(\mu \mathrm{m})$

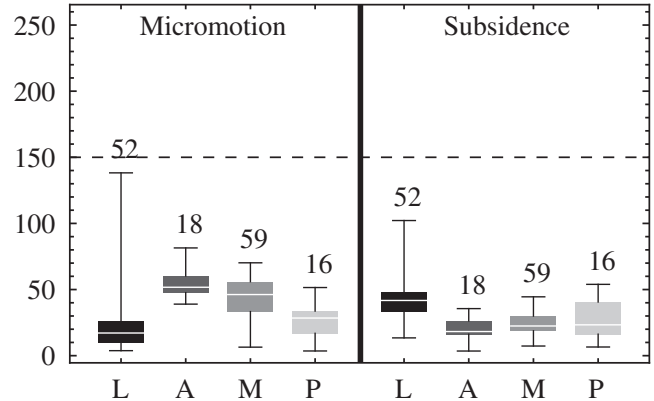

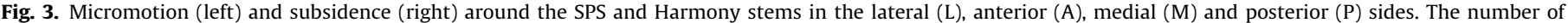
samples per side are indicated. The dotted horizontal line corresponds $150 \mu \mathrm{m}$. 

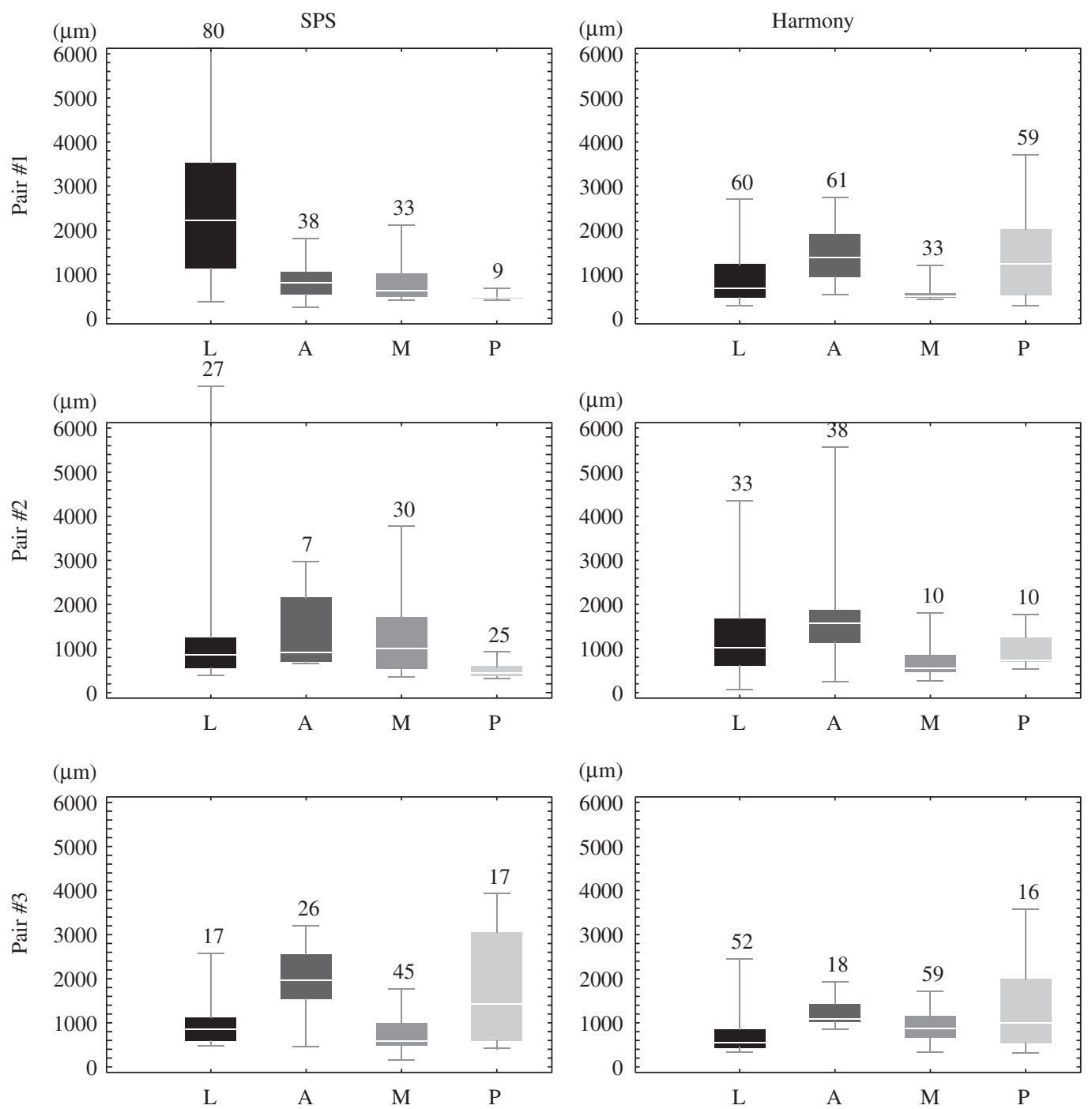

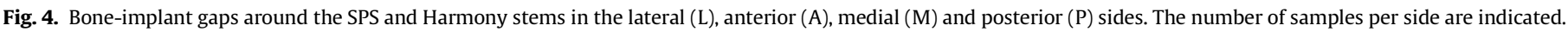

the strain distribution on the stem surfaces was higher in the medial and anterior side.

\section{Discussion}

The initial stability of cementless femoral components used in total hip arthroplasty is a key condition for the long-term survival of the implant. It is indeed widely accepted that a lack of stability prevents bone ingrowth, but promotes an interfacial layer of fibrous tissue (Pilliar et al., 1986; Engh et al., 1992; Bragdon et al., 1996; Jasty et al., 1997). A relevant measurement of this stability is currently only feasible on cadaveric models. In our study, we propose to use a new method for measuring the periimplant micromotion, subsidence and gap, and for estimating the strain interstitial. The second aim of this study was to apply this technique to in a paired comparison of two typical stem designs and to evaluate their relative stability.

The initial experimental method presented in a previous technical paper was successfully extended to get a large number of measurement points over the stem surface (Gortchacow et al., 2011). The local three-dimensional micromotion measurement was also extended with a measurement of the bone-stem gap, which was used to estimate the strain within the interstitial tissue. Overall, the measured micromotion was within a physiological range and below the critical value of $150 \mu \mathrm{m}$, except for one case (SPS in pair \#1). Subsidence values were also coherent with typical reported data in cadaveric or clinical studies, except for the same case, which explains its high micromotion. From a clinical point of view, the subsidence for the SPS stem of pair \#1 was not acceptable, but is however observed in real clinical situations and requires a revision (Flecher et al., 2010). This large subsidence was curtained because of the shape of the medullary canal, not adapted for an anatomical stem. In pair \#2 and \#3, micromotion was notably higher in the anterior and medial sides than in the posterior and lateral sides. This can be related to the application point of the load (the femoral head center), which is on the medial side of the stem axis. Such a localized behavior was not observed with the strain. The difference between micromotion and strain reveals the importance of accounting not only for micromotion, but also for peri-implant gap. Strain within the interstitial tissue indeed strongly depends on this gap, and is known to affect its transformation into a healthy bone tissue.

The comparison of the two stem designs (for pair \#2 and \#3) in terms of micromotion showed that the straight stem was more adapted to the anatomy in pair \#3, however the micromotion was not too far above the critical limit with the anatomical stem. When we compare the interstitial strain, again with pair \#2 and 

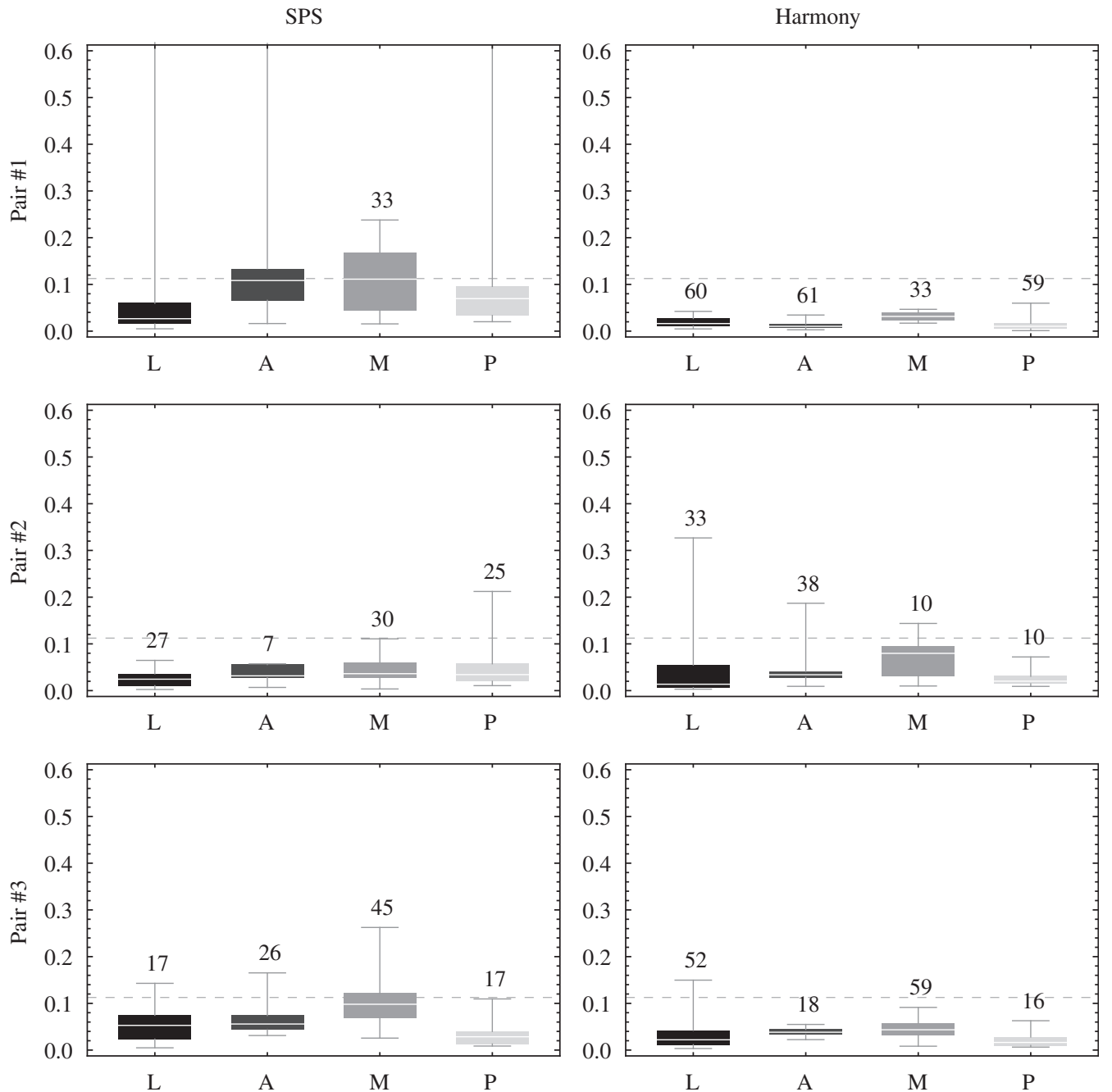

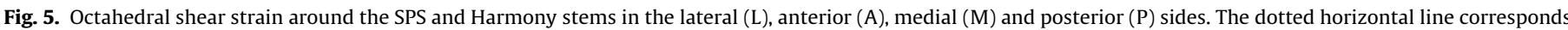
a strain of $11 \%$.

\#3, there was a slight advantage for the SPS in pair \#2, but conversely strain was lower with the Harmony in pair \#3. Our results did not identify the best stem, but rather suggest that that the choice of an anatomic or straight stem should be made according to the shape of the medullary canal. A "champagneflute" morphology is more adapted for an anatomic stem, whereas a "stove-pipe" morphology will do better with a straight stem (Noble et al., 1988). The femurs of pair \#2 were indeed more straight shaped, promoting a straight design of the stem, while femurs of pair \#3 were more tulip-like shaped, suggesting a better initial stability of an anatomical design.

The multiple and simultaneous measurements of peri-implant micromotion, subsidence and gap presented in this paper are unique. They are based on a local measurement and provide an estimate of the of interstitial tissue strain. Micromotion has already been measured on cadaveric femurs, but only on a limited number of simultaneous locations. The values of micromotion reported here are compatible with other cadaveric measurements (Walker et al., 1987; Gilbert et al., 1992; Buhler et al., 1997; Britton et al., 2004). Although the comparison is not always easy because of different techniques and loading conditions, most cadaveric studies report maximal amplitude of micromotion and subsidence of about $100 \mu \mathrm{m}$, with higher values on the medial than on the lateral side.

The strength of the present study consists in simultaneously measuring peri-implant micromotion, subsidence and gap in a large number of points on the implant surface, without assuming bone as a rigid body. With this method, we can extend the motion measurement to estimate the strain with the interstitial tissue, which is known to be a key parameter for the mechano-transduction phenomenon at the cellular level (Davies, 2003). Although this interstitial tissue was not present in our cadaveric study, it is so soft compared to the bone and the implant that it would not contribute the mechanical stability of the implant. Thanks to the custom-made loading device, this measurement could be made with rather high loading amplitudes. The maximal loading amplitude corresponded approximately to 2.5 times the body weight and was associated to walking, according to experimental measurement with an instrumented prosthesis (Bergmann et al., 2001). The loading amplitude of $2000 \mathrm{~N}$ was used for all femurs, since we had no information on donor weight. Our measurement technique would also applicable to $\mathrm{CoCr}$ stems, but we have not evaluated the effect of CT artifact, which are known to be higher than for titanium stems.

This experimental setup had also some limitations. In the current experimental setup the muscle force is not included. Adding the abductor muscle force was however reported to reduce slightly the micromotion measurement (Britton et al., 2003; Park et al., 2010). Although it would be more difficult to add the muscle force in our system compared to LVDTs devices, it is still feasible. In our study, the load was only applied once, but it 
was reported that micromotion and subsidence increase during the first 100 cycles (Britton et al., 2004). Another limitation concerns the number and distribution of markers, and measuring points. Although we have much more measurement sites than all other experimental methods, there is still a limitation in the spatial distribution of the markers. The minimal distance between two markers is indeed constrained by the size of the markers and the resolution of the $\mu \mathrm{CT}$ measure, which is also contained by the scanning time. We also were confronted to the problem of having a more uniform distribution of the markers on the bone surface, which is still an issue to improve in the future. Bone markers were press fitted into the bone surface of the reamed cavity, but a few of them were too close to the implant and displaced with it instead of staying on the bone surface. These bone markers were easily identified and were not considered in the micromotion analysis. In the present study, the micromotion error was higher than the error reported by other studies using LVDTs. However, our error of $15 \mu \mathrm{m}$ was small enough to characterize potentially loose implants having micromotion above $150 \mu \mathrm{m}$. Most importantly, the small error reported by LDVT's devices do not account for the bias caused by deformation of the measuring system itself (Tarala et al., 2009). Assuming a gap error of $300 \mu \mathrm{m}$ and the measure error in micromotion of $15 \mu \mathrm{m}$ in all orthogonal directions, we estimated the strain error using error propagation reduced to quadratic terms. The median of the strain error distribution over all measurements was 0.01 , which remains reasonable compare to the critical stain value of 0.11 . Finally, this study was weakened by the limited by the number of specimens, as the lack of information on the donors. Although we have no statistical significance, we think that these two different designs should be chosen according to the patient's femur shape, which is actually the common surgical planning practice. One more problem is the age of the donors, which is related to a significant osteopenia. This induces a more "stovepipe"-like shape of the medullary canal than in younger people, which may also change the results (Noble et al., 1995).

To conclude, this study reveals the strength of this new method and the importance of measuring peri-implant micromotion and gap, in multiple and simultaneous sites, for analyzing the initial stability of cementless implants. It is indeed accepted that the initial stability of cementless implants is a key condition for the long-term success of these implants. Moreover, since that fixation of the stem results of its complex 3D mechanical support, we have showed the importance of measuring these values overall around the stem rather than only in a few points. This new experimental method for measuring micromotion and gap around bone implants could thus be applied to compare the primary stability of stem designs, or surgical techniques, but could also be used to validate numerical models predicting this primary stem stability.

\section{Conflict of interest statement}

\section{None of the authors has any conflict of interest.}

\section{Acknowledgments}

This research was supported by a grant from the Swiss National Science Foundation (SNSF No. 125498) and by the Center of Translational Biomechanics EPFL-CHUV-DAL. The authors thank Symbios Orthopédie SA (Yverdon-Les-Bains, Switzerland) for donating implants and Philippe Chavanne (R\&D director of the hip division at Symbios) for technical assistance.

\section{References}

Baleani, M., Cristofolini, L., Toni, A., 2000. Initial stability of a new hybrid fixation hip stem: experimental measurement of implant-bone micromotion under torsional load in comparison with cemented and cementless stems. Journal of Biomedical Materials Research 50 (4), 605-615.

Bergmann, G., Deuretzbacher, G., Heller, M., Graichen, F., Rohlmann, A., Strauss, J., Duda, G.N., 2001. Hip contact forces and gait patterns from routine activities. Journal of Biomechanics 34 (7), 859-871.

Bragdon, C.R., Burke, D., Lowenstein, J.D., O'Connor, D.O., Ramamurti, B., Jasty, M., Harris, W.H., 1996. Differences in stiffness of the interface between a cementless porous implant and cancellous bone in vivo in dogs due to varying amounts of implant motion. Journal of Arthroplasty 11 (8), 945-951.

Britton, J.R., Lyons, C.G., Prendergast, P.J., 2004. Measurement of the relative motion between an implant and bone under cyclic loading. Strain 40 (4), 193-202.

Britton, J.R., Walsh, L.A., Prendergast, P.J., 2003. Mechanical simulation of muscle loading on the proximal femur: analysis of cemented femoral component migration with and without muscle loading. Clinical Biomechanics (Bristol, Avon) 18 (7), 637-646.

Buhler, D.W., Oxland, T.R., Nolte, L.P., 1997. Design and evaluation of a device for measuring three-dimensional micromotions of press-fit femoral stem prostheses. Medical Engineering \& Physics 19 (2), 187-199.

Callaghan, J.J., Fulghum, C.S., Glisson, R.R., Stranne, S.K., 1992. The effect of femoral stem geometry on interface motion in uncemented porous-coated total hip prostheses. Comparison of straight-stem and curved-stem designs. The Journal of Bone and Joint Surgery American 74 (6), 839-848.

Cristofolini, L., Teutonico, A.S., Monti, L., Cappello, A., Toni, A., 2003. Comparative in vitro study on the long term performance of cemented hip stems: validation of a protocol to discriminate between "good" and "bad" designs. Journal of Biomechanics 36 (11), 1603-1615.

Cristofolini, L., Varini, E., Viceconti, M., 2007. In-vitro method for assessing femoral implant-bone micromotions in resurfacing hip implants under different loading conditions. Proceedings of Institution Mechanical Engineering H 221 (8), 943-950.

Davies, J.E., 2003. Understanding peri-implant endosseous healing. Journal of Dental Education 67 (8), 932-949.

Engh, C.A., O'Connor, D., Jasty, M., McGovern, T.F., Bobyn, J.D., Harris, W.H., 1992. Quantification of implant micromotion, strain shielding, and bone resorption with porous-coated anatomic medullary locking femoral prostheses. Clinical Orthopaedics and Related Research 285, 13-29.

Flecher, X., Pearce, O., Parratte, S., Aubaniac, J.-M., Argenson, J.-N., 2010. Custom cementless stem improves hip function in young patients at 15-year followup. Clinical Orthopaedics and Related Research 468 (3), 747-755.

Gheduzzi, S., Miles, A.W., 2007. A review of pre-clinical testing of femoral stem subsidence and comparison with clinical data. Proceedings of Institution Mechanical Engineering H 221 (1), 39-46.

Gilbert, J.L., Bloomfeld, R.S., Lautenschlager, E.P., Wixson, R.L., 1992. A computerbased biomechanical analysis of the three-dimensional motion of cementless hip prostheses. Journal of Biomechanics 25 (4), 329-340.

Gortchacow, M., Wettstein, M., Pioletti, D.P., Terrier, A., 2011. A new technique to measure micromotion distribution around a cementless femoral stem. Journal of Biomechanics 44 (3), 557-560.

Hozack, W.J., Rothman, R.H., Booth Jr., R.E., Balderston, R.A., 1993. Cemented versus cementless total hip arthroplasty. A comparative study of equivalent patient populations. Clinical Orthopaedics and Related Research 289, 161-165.

Huiskes, R., Van Driel, W.D., Prendergast, P.J., Soballe, K., 1997. A biomechanical regulatory model for periprosthetic fibrous-tissue differentiation. Journal of Materials Science: Materials in Medicine 8 (12), 785-788.

Jasty, M., Bragdon, C., Burke, D., O'Connor, D., Lowenstein, J., Harris, W.H., 1997. In vivo skeletal responses to porous-surfaced implants subjected to small induced motions. Journal of Bone \& Joint Surgery-American 79 (5), 707-714.

Mäkelä, K.T., Eskelinen, A., Paavolainen, P., Pulkkinen, P., Remes, V., 2010. Cementless total hip arthroplasty for primary osteoarthritis in patients aged 55 years and older. Acta Orthopaedica 81 (1), 42-52.

Merle, C., Clarius, M., Aldinger, P.R., 2010. Long-term results of uncemented stems in total hip arthroplasty: analysis of survival rates with a minimum 15-year follow-up. Orthopade 39 (1), 80-86.

Noble, P.C., Alexander, J.W., Lindahl, L.J., Yew, D.T., Granberry, W.M., Tullos, H.S., 1988. The anatomic basis of femoral component design. Clinical Orthopaedics and Related Research 235, 148-165.

Noble, P.C., Box, G.G., Kamaric, E., Fink, M.J., Alexander, J.W., Tullos, H.S., 1995. The effect of aging on the shape of the proximal femur. Clinical Orthopaedics and Related Research 316, 31-44.

Park, Y., Albert, C., Yoon, Y.S., Fernlund, G., Frei, H., Oxland, T.R., 2010. The effect of abductor muscle and anterior-posterior hip contact load simulation on the invitro primary stability of a cementless hip stem. Journal of Orthopaedic Surgery and Research 5, 40.

Pilliar, R.M., Lee, J.M., Maniatopoulos, C., 1986. Observations on the effect of movement on bone ingrowth into porous-surfaced implants. Clinical Orthopaedics and Related Research 208, 108-113.

Riddle, R.C., Donahue, H.J., 2009. From streaming potentials to shear stress: 25 Years of bone cell mechanotransduction. Journal of Orthopaedic Research 27 (2), 143-149. 
Soballe, K., Hansen, E.S., Brockstedt-Rasmussen, H., Bunger, C., 1993. Hydroxyapatite coating converts fibrous tissue to bone around loaded implants. Journal of Bone \& Joint Surgery 75 (2), 270-278.

Steimer, O., Adam, F., Johann, S., Pape, D., 2006. Primary stability of cementless implanted hip stems made of titanium alloy with metaphyseal fixation. A prospective clinical roentgen-stereometry-analysis (RSA) study. Zeitschrift Fur Orthopadie Und Ihre Grenzgebiete 144 (6), 587-593.
Tarala, M., Janssen, D., Verdonschot, N., 2009. Typical experimental methods do not capture micromotion at the implant-bone interface. In: Proceedings of the 55th Annual Meeting of the Orthopaedic Research Society, Las Vegas.

Walker, P.S., Schneeweis, D., Murphy, S., Nelson, P., 1987. Strains and micromotions of press-fit femoral stem prostheses. Journal of Biomechanics 20 (7), 693-702. 